\title{
Utility of 3D printing of left atrial appendages for closure with Watchman Devices and comparison of computed tomography and transesophageal echocardiography based models
}

\author{
Mohammed M. Ansari MD, Ali Akbar Arvandi MD, Ronnie Orozco MS, CCR, Carlos Morales MD, \\ Kate M. Serralde C-CM, Bernardo Galvan BS, Ozman Ochoa BS MS, Katherine G. Holder BBA, \\ Marina Iskandir MD, Scott Shurmur MD
}

\begin{abstract}
Three-dimensional (3D) printed cardiac models are useful for WATCHMAN device procedural planning, sizing, and complication reduction. These models also provide accurate representation of dynamic heart anatomy, helping practitioners determine their procedural approach and select proper device sizing. While the advantages of 3D models obtained from computed tomography (CT) and transesophageal echocardiography (TEE) over 2D TEE imaging for WATCHMAN procedural planning has been demonstrated, this project aims to directly compare $3 D C T$ models and $3 D$ TEE models to determine which model provides more useful information. We used CT and TEE 2D imaging studies from patients who underwent left atrial appendage (LAA) WATCHMAN closure device implantation as templates for $3 D$ cardiac models. These $3 D$ models were scored using a 10-point Likert questionnaire by a diverse team that included cardiologists, research specialists, medical students, and 3D printing technicians. Three-dimensional models developed from CT scans demonstrated better value than TEE 3D models by all qualitative measures. Scoring indicates that CT based 3D models are superior tools for WATCHMAN sizing, multilevel medical education, and physician preparation. To our knowledge, this is the only study that compares 3D models crafted from each imaging modality, and we suggest that future use of $3 D$ modeling techniques should be based on CT scans.
\end{abstract}

Keywords: cardiac three-dimensional models, three-dimensional printing, left atrial appendage occlusion, computed tomography, transesophageal electrocardiogram, WATCHMAN Closure Device, medical education

\section{INTRODUCTION}

The creation of three-dimensional (3D) organ models, specifically of the heart and associated pathologies, has resulted in multiple educational and clinical applications. For example, 3D cardiac models

Corresponding author: Mohammed Ansari Contact Information: Mac.Ansari@ttuhsc.edu DOI: $10.12746 /$ swrccc.v9i37.789 have been used in both undergraduate and resident medical education. One comparative study reported improved learning and experience outcomes in undergraduate medical students regarding heart anatomy and congenital heart disease when 3D printed models were used. ${ }^{1}$ In addition, residents have demonstrated an increase in clinical skill using 3D models for learning and have shown an increased ability in describing and communicating treatment plans to patients and their own teams. ${ }^{2,3}$ The use of 3D cardiac models for describing care to patients has also been helpful in 
obtaining informed patient consent. ${ }^{3}$ Due to the complexity and variability of an individual's heart and associated vessels, 3D printed models acquired from imaging data points have allowed physicians to better plan and prepare for complex procedures. ${ }^{3}$

The use of 3D printed cardiac models has improved the planning and practicing for intricate procedures, such as the WATCHMAN (Boston Scientific Left Atrial Appendage Closure Implant) implantation, which is the focus of this study. The interventionist must deploy properly sized WATCHMAN devices to decrease the risk of surgical complications, including pericardial effusions, appendage wall perforations, and device-related thrombus. ${ }^{4}$ Three-dimensional printing can better present the anatomy to the clinician when planning a surgery or procedure. ${ }^{5}$ In addition, current cardiovascular surgical training is mostly opportunity based, which limits uniform exposure of the trainees to various procedures. ${ }^{3}$ Therefore, 3D cardiac models have an increasingly significant role in preparing medical students, residents, and fellows for these intricate procedures through practicing on varying pathological presentations. ${ }^{3}$

Three-dimensional models obtained from computed tomography (CT) and transesophageal echocardiography (TEE) data points have had their utility compared to traditional imaging methods for left atrial appendage (LAA) WATCHMAN closure device implantation. Threedimensional printed heart models produced from CT imaging data points have an increased rate of correct device sizing prediction and implantation compared to TEE imaging. ${ }^{6,7}$ Transesophageal echocardiography images of LAA have also been translated into 3D models and have decreased procedure times, deployment attempts, device resizing, complications, risk of stroke, and peridevice leakage and increased the overall success rate compared to devices implanted using only TEE guided imaging. ${ }^{4}$ Although $3 \mathrm{D}$ models created from CT scans must include radiation exposure, procedural planning of WATCHMAN implantation using these models in comparison to TEE image guided planning has improved procedure performance and occluder accuracy, decreasing the exchange of devices and catheters, thus creating simpler and safer cases. ${ }^{6}$ While both 3D models of CT and TEE data points have been compared to traditional TEE image guided procedure planning, there is limited literature comparing $3 \mathrm{D}$ printed CT models with 3D printed TEE models in LAA WATCHMAN device planning. ${ }^{6,7,4}$

Given the relative novelty of 3D modeling in preoperative LAA occlusion, the current clinical studies have two main goals. First, there is a need for larger cohort studies to validate the available results showing significantly improved work efficiency and outcomes in LAA WATCHMAN occlusion procedures using 3D modeling. Second, there should be direct comparison of the prediction of WATCHMAN device size with the different image acquisition approaches. ${ }^{6}$ The specific aim of our study is to compare 3D TEE models and 3D CT models to determine which method provides superior modeling. Our hypothesis is that 3D CT images will provide a more accurate model since $2 \mathrm{D}$ measurements have been close to exact measurements taken in vivo during procedures.

\section{Methods}

\section{Study Design}

A retrospective study of patients was completed using pre-procedural imaging. Our subjects included men and women treated at our hospital (University Medical Center, Lubbock, Texas) between 2017 and 2019. Five patients were selected who had simultaneously undergone CT and TEE prior to the procedure. This criterion was used to rule out time related bias in the images and assure equal hemodynamics during image acquisition for both imaging modalities. All five patients were adequately hydrated prior to the imaging studies and procedure. The patients selected had atrial fibrillation, were previously on anticoagulation therapy, and were considered appropriate candidates for LAA WATCHMAN closure device implantation. The anatomy in the WATCHMAN procedure involves both complexity and individuality, and a complete understanding of the morphology, structure, and size of the LAA is required before the operation. ${ }^{8}$ Given the intricacy, LAA occlusion was considered a good procedure on which to base our comparison of $3 \mathrm{D}$ print models. Using $2 \mathrm{D}$ images from each of the five patients' CT and TEE scans, 3D cardiac models were printed post-procedurally. The 3D CT and 3D TEE models were then compared using qualitative analysis based on a 10-point Likert questionnaire to determine which provided better information. The Likert 


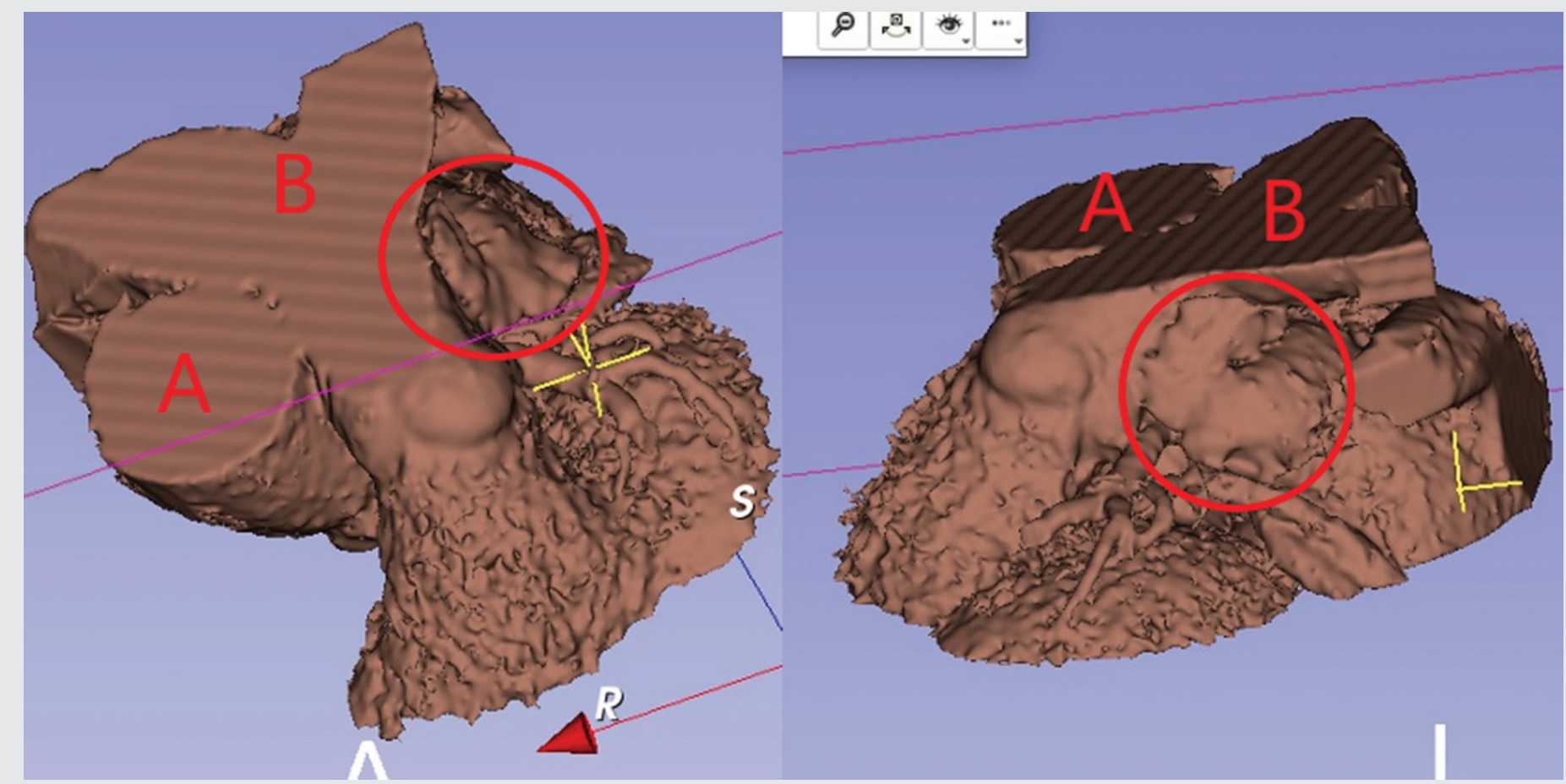

Figure 1. Left: Superior view of a heart slice from CT acquired data. The red circle outlines the left atrial appendage as seen from this view. A. depicts the aorta. B. depicts the bifurcation of the pulmonary artery. Right: Lateral view of same heart slice. Superimposed demarcations represent the same anatomical structures as left image.

questionnaire included questions involving the potential impact 3D printed models could have on medical education, patient understanding, and physician preoperative planning. Seven volunteers from our institution with varying backgrounds participated in the study. Each volunteer was given the opportunity to examine the 3D CT and 3D TEE models with two copies of the questionnaire to evaluate both models simultaneously. Participants included an interventional/structural cardiologist, a cardiologist specializing in cardiac imaging, a cardiology fellow, a cardiovascular cardiology research coordinator, two medical students, and a 3D printer technician with a background in art history and sculpture. All professionals had more than 3 years' experience in their fields. The two medical students underwent specific training before evaluating the models.

\section{IMAGE ACQUISITION, PROCESSING, AND PRINTING}

Computed tomography and TEE images were imported into an open source editing and segmentation software (3D Slicer version 4.10.2; www.slicer.org) program. The software generated a geometric surface mesh model (Figure 1) that underwent noise filtration prior to printing. The digital models were exported as low force stereolithography files and printed using a $250 \mathrm{~mW}$ laser power optic engine printer (Formlabs Form 3; Somerville, MA) (Figure 2). A pliable media (Formlabs Durable Resin) was layered at a height of 100 microns to more closely resemble cardiac tissue (Figures 3 and 4).

\section{RESULTS}

The 3D CT model was preferred over the 3D TEE models across all measured variables (Table). All models were printed and questionnaires were completed successfully. The total scores from the Likert Scale average of all 7 judges for the CT 3D model was 9.00 , which was substantially greater than the 3D TEE models average of 2.16. On the question about size accuracy, the CT model scores averaged 8.81 , and the TEE model 


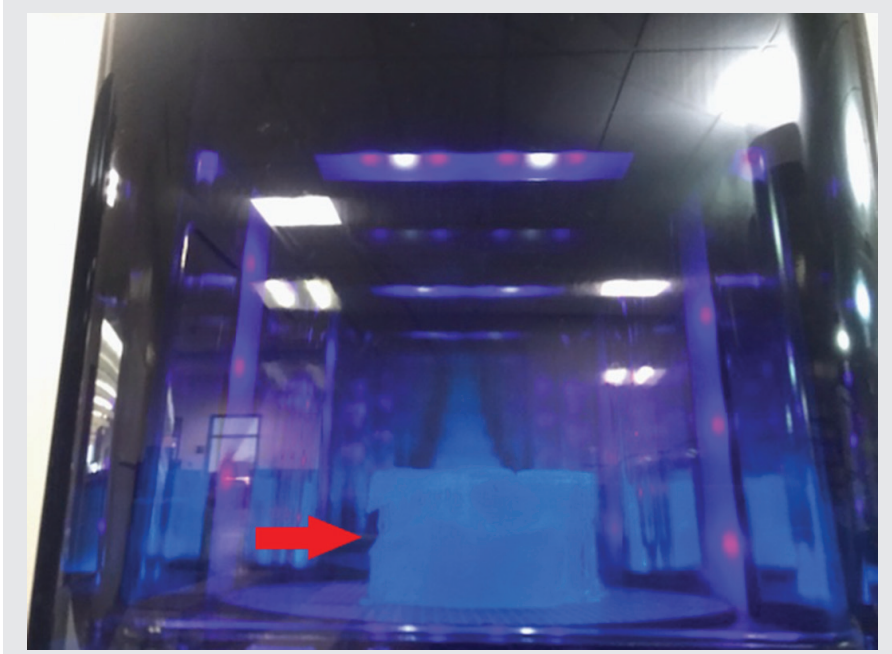

Figure 2. Close up of a 3D printer with a print in progress. Arrow depicts a printed model prior to removal of excess material.

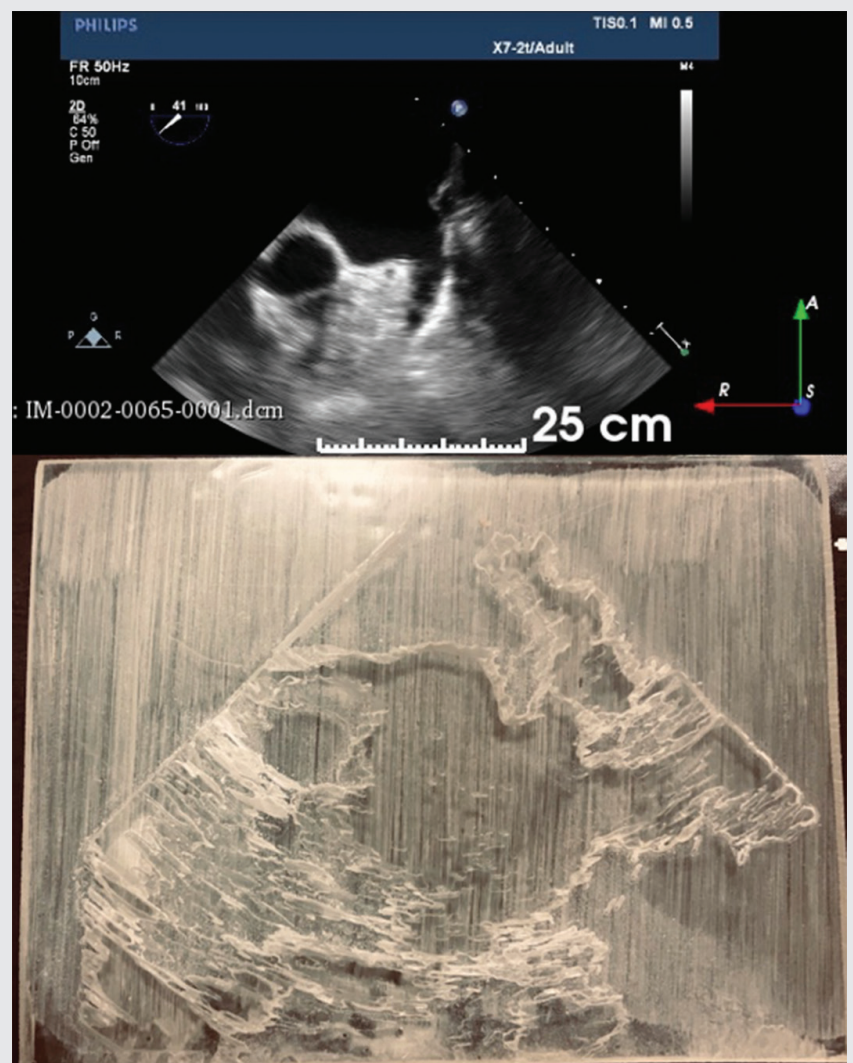

Figure 3. Top: TEE acquired image of left atrium. Bottom: 3D TEE printed model.

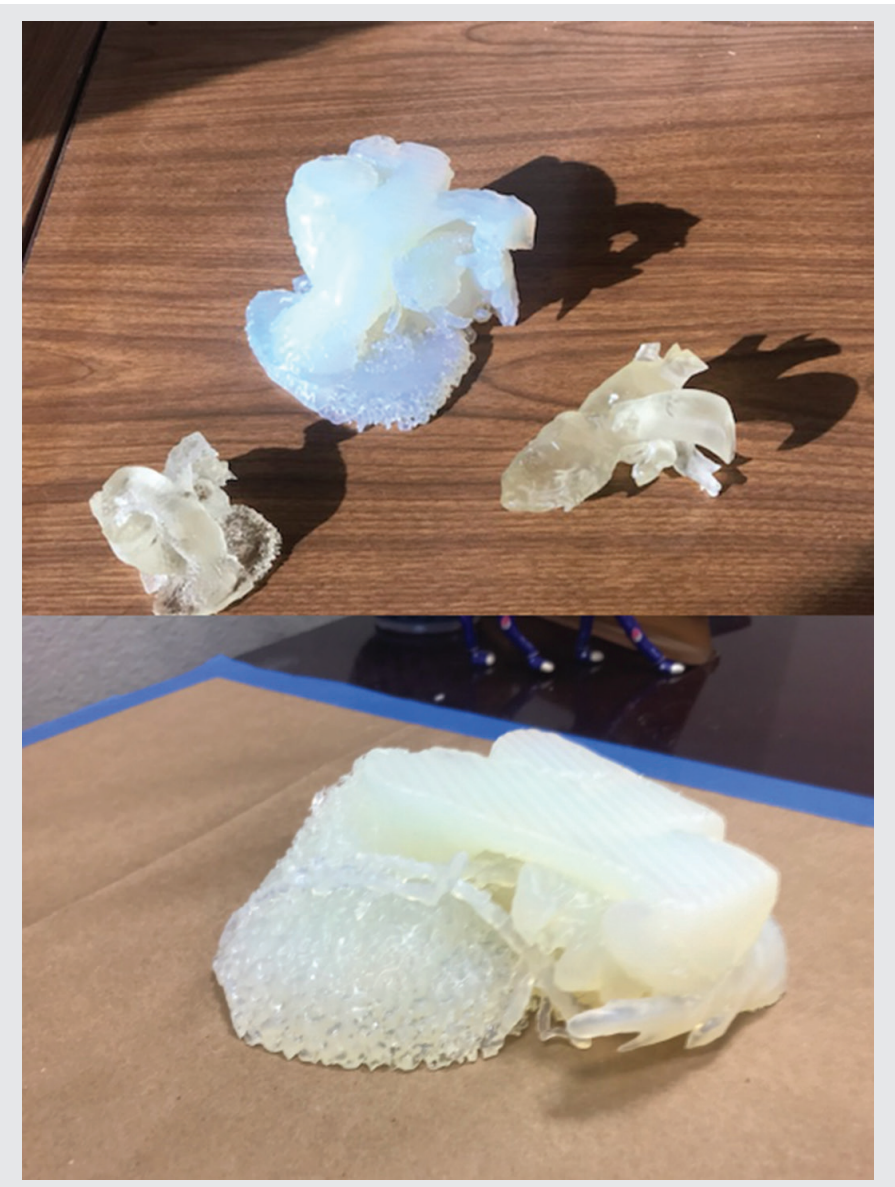

Figure 4. Top: Multiple prototype 3D CT models of varying scale and materials. Bottom: A final 3D CT model used for qualitative assessment.

scores averaged 1.96. Regarding shape accuracy, CT models averaged 8.88; TEE models averaged 1.80. The overall educational quality average was 9.04 for CT models and 3.56 for TEE models. When judging ability to influence procedural planning, CT models averaged 9.08, and TEE models averaged 1.80. Averages for ability to size devices preoperatively were 9.04 for CT models and 1.48 for TEE models.

It was more challenging to convert images from echocardiography to $3 \mathrm{D}$ prints than $\mathrm{CT}$ images, and it took less time to print the CT based models than ultrasound based models. Printing CT based 3D models required $20 \pm 4$ hours; printing ultrasound based models required $30 \pm 5$ hours. 
Table. Average Likert Scale Scores for the 3D CT Models and 3D TEE Models

\begin{tabular}{|c|c|c|}
\hline \multicolumn{3}{|l|}{ Likert Scores } \\
\hline & $\begin{array}{l}\text { CT Based } \\
\text { Model }\end{array}$ & $\begin{array}{c}\text { TEE Based } \\
\text { Model }\end{array}$ \\
\hline Size accuracy & $8.88 \pm 1.11 *$ & $1.96 \pm 1.73$ \\
\hline Shape accuracy & $8.88 \pm 0.99$ & $1.80 \pm 1.42$ \\
\hline Realism & $8.80 \pm 1.05$ & $1.52 \pm 1.53$ \\
\hline Educational quality & $9.04 \pm 0.97$ & $3.56 \pm 3.70$ \\
\hline $\begin{array}{l}\text { Quality as a tool for } \\
\text { patient education and } \\
\text { informed consent }\end{array}$ & $9.04 \pm 0.93$ & $2.96 \pm 3.41$ \\
\hline Structural accuracy & $9.00 \pm 0.92$ & $1.68 \pm 1.51$ \\
\hline $\begin{array}{l}\text { Ability to influence } \\
\text { procedural planning }\end{array}$ & $9.08 \pm 0.93$ & $1.80 \pm 1.60$ \\
\hline $\begin{array}{l}\text { Differentiating color } \\
\text { quality }\end{array}$ & $9.00 \pm 0.82$ & $3.32 \pm 3.74$ \\
\hline $\begin{array}{l}\text { Ability to size devices } \\
\text { pre-operatively }\end{array}$ & $9.04 \pm 0.88$ & $1.48 \pm 1.38$ \\
\hline $\begin{array}{l}\text { Decrease consultation } \\
\text { time without } \\
\text { compromising patient } \\
\text { understanding }\end{array}$ & $9.00 \pm 0.88$ & $1.48 \pm 1.32$ \\
\hline Overall average & 9.00 & 2.16 \\
\hline
\end{tabular}

\section{Discussion}

This retrospective study compares $3 \mathrm{D}$ printed models obtained from CT and TEE imaging for patients with an LAA who met the inclusion criteria to determine the preferred method for WATCHMAN device procedural planning, anatomy presentation, and medical education. Judges were recruited whose expertise in cardiology, imaging, and artistry helped determine that the 3D CT LAA model provided a more accurate depiction of heart anatomy and better approximation of WATCHMAN device sizing and implantation than the 3D TEE model. While previous studies have demonstrated the efficacy of 3D models over standard imaging approaches, this project aimed to determine which 3D print would provide more accurate measurements: CT vs TEE. Since CT scans have better image acquisition, this project's hypothesis was that the 3D CT model would provide more information than the 3D TEE model.

Three-dimensional printed cardiovascular models have important clinical and educational benefits, including more efficient planning of procedures, more accurate depiction of heart anatomy and vasculature dynamics for educational purposes, and opportunities to practice interventions with different pathologies. ${ }^{3}$ Previous studies have also compared the utility of $3 \mathrm{D}$ printed models to traditional TEE imaging for LAA for WATCHMAN device sizing and insertion and have demonstrated that both TEE and $\mathrm{CT}$ acquired $3 \mathrm{D}$ models provide more accurate procedural planning and device sizing than the $2 \mathrm{D}$ image ${ }^{4,6,7}$ Based on the scores provided by the project's judges, a 3D CT model provides a more accurate depiction of heart anatomy for pre-procedural planning, patient education, and medical education. However, it was hoped that the 3D TEE would have comparable results due to the decreased cost of imaging and absence of radiation exposure compared to CT scans. ${ }^{9}$ Nonetheless, the 3D CT prints had higher scores across all categories of evaluation and was therefore considered more informative.

There are important advantages and disadvantages with CT 3D models. Computed tomography scans have increased cost and require significant radiation exposure, which may be undesirable in some patients. Computed tomography imaging also requires the administration of contrast which limits its use in older patients with poor renal function. However, the detailed image and models produced from CT scans provide more information about dynamic heart anatomy, improve procedure planning, and have more utility in medical education. It is also important to note the CT acquisition possesses less risk for complications, especially in patients with a risk for or a history of esophageal varices.

There are also advantages and disadvantages with TEE 3D models. One major drawback to TEE based 3D models compared to those from CT scans is the compromised image and model detail. When comparing the two modalities, TEE 3D models are not as useful in understanding the heart anatomy, procedure planning, or in medical education. However, in situations in which exposure to radiation from CT scans may not be appropriate, TEE based models may be suitable. Transesophageal echocardiography 3D models also have advantages in portability, 
accessibility at most healthcare centers, high temporal resolution, and no radiation exposure. ${ }^{9}$

\section{LIMITATIONS}

Our study results were entirely qualitative, and no quantitative data were collected. We used a small group of healthcare professionals to evaluate the models. In future studies, we recommend comparing 3D CT and 3D TEE images with quantitative measures to remove any possibility of bias. If a repeat qualitative study is performed, we recommend a larger group of evaluators. Additionally, we recommend including an analysis of patient complications with and without 3D models.

\section{ConcLusion}

The 3D models obtained from CT scans were better than the 3D models obtained from TEE for depicting LAA anatomy and WATCHMAN device sizing. By recruiting expert judges with applicable professional backgrounds, the utility of both types of 3D models was determined through Likert scale assessments.

\section{ACKNowledgements}

Special thanks to Smiley Garcia, Kelsey Marks, and Jill Holden for their help and support. This study was conducted with funding from the $\mathrm{CH}$ Foundation, Lubbock, Texas.

Article citation: Ansari MM, Arvandi AA, Orozco R, Morales C, Serralde KM, Galvan B, Ochoa O, Holder KG, Iskandir M, Shurmur S. Utility of 3D printing of left atrial appendages for closure with Watchman Devices and comparison of computed tomography and transesophageal echocardiography based models. The Southwest Respiratory and Critical Care Chronicles 2021;9(37):60-65 From: Department of Internal Medicine, Texas Tech University Health Sciences Center, Lubbock, Texas Submitted: $12 / 17 / 2020$

Accepted: $1 / 12 / 2021$

Reviewer: Ankush Lahoti MD

Conflicts of interest: none

This work is licensed under a Creative Commons Attribution-ShareAlike 4.0 International License.

\section{REFERENCES}

1. Su W, Xiao Y, He S, Huang P, et al. Three-dimensional printing models in congenital heart disease education for medical students: A controlled comparative study. BMC medical education 2018;18(1):178. https://search.datacite.org/works/10.1186/ s12909-018-1293-0. doi: 10.1186/s12909-018-1293-0.

2. Loke Y, Harahsheh AS, Krieger A, et al. Usage of 3D models of tetralogy of Fallot for medical education: Impact on learning congenital heart disease. BMC medical education 2017; 17(1):54.

3. Giannopoulos AA, Mitsouras D, Yoo SJ, et al. Applications of 3D printing in cardiovascular diseases. Nat Rev Cardiol 2016; 13(12):701-718.

4. Fan Y, Yang F, Cheung GS, et al. Device sizing guided by echocardiography-based three-dimensional printing is associated with superior outcome after percutaneous left atrial appendage occlusion. J Amer Soc Echocardiography 2019;32(6):708-719.

5. Abudayyeh I, Gordon B, Ansari MM, et al. A practical guide to cardiovascular $3 \mathrm{D}$ printing in clinical practice: Overview and examples. J Interv Cardiol 2018;31(3):375-383.

6. Hell M, Achenbach S, Yoo I, et al. 3D printing for sizing left atrial appendage closure device: Head-to-head comparison with computed tomography and transoesophageal echocardiography. Euro Intervention 2017;13(10):1234-1241. https://search.datacite.org/ works/10.4244/eij-d-17-00359. doi: 10.4244/eij-d-17-00359.

7. Eng MH, Wang DD, Greenbaum AB, et al. Prospective, randomized comparison of 3-dimensional computed tomography guidance versus TEE data for left atrial appendage occlusion (PRO3DLAAO). Catheter Cardiovasc Interv 2018;92(2): 401-407.

8. Li H, Qingyao, Bingshen, et al. Application of 3D printing technology to left atrial appendage occlusion. Int J Cardiol 2017; 231:258-263.

9. Liu P, Liu R, Zhang Y, et al. The value of 3D printing models of left atrial appendage using real-time 3D transesophageal echocardiographic data in left atrial appendage occlusion: Applications toward an era of truly personalized medicine. Cardiology 2016;135(4):255-261. 